\title{
Sloped-Gate Voltage Method for Improving Measurement of Poly-Si Nanowire FET in Aqueous Environment
}

\author{
Chien-Hung Chen, ${ }^{\dagger}{ }^{\dagger}$ Yi-Jr Su, ${ }^{\S}$ Chih-Heng Lin, ${ }^{\dagger}$ Tai-Shan Liao, ${ }^{\ddagger}$ Yuh-Shyong Yang, ${ }^{*} \dagger$ \\ and Chi-Hung Hwang* $*$ \\ ${ }^{\dagger}$ Institute of Biological Science and Technology, National Chiao Tung University, Hsinchu 300, Taiwan \\ ${ }^{\ddagger}$ Instrument Technology Research Center, National Applied Research Laboratories, Hsinchu 300, Taiwan \\ ${ }^{\S}$ National Synchrotron Radiation Research Center, Hsinchu 300, Taiwan
}

Supporting Information

ABSTRACT: Nanowire field-effect transistors are suited to study the activity of biomolecules in bionanotechnology. The changes of biomolecules process are efficiently affected the charge at the nanowire surface; thus, the electrical characterization of NW-FET is changed. Although NW-FET is a wellknown device in bioapplications, however, the intrinsic electrical characterization of NW-FET effect on real electrical measurement is not well studied. We present herein a novel measurement method to avoid errors in electrical characteristic of nanowire field-effect transistors. A physical model is

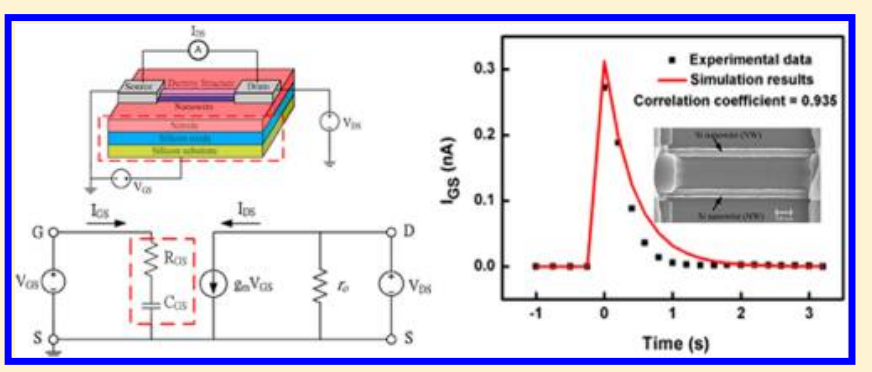
developed to explore the effect of the leakage current, which is influenced by the charging effect of an equivalent capacitor in a NW-FET. We also present a sloped-gate voltage method to reduce the effect of equivalent capacitor in air, liquid, and phosphate buffered solution. The application of the sloped-gate voltage method significantly increases the stability of electrical characterization measurements. This method can also be easily applied to biosensing experiments.

\section{INTRODUCTION}

Biological sensors based on semiconductor nanowire field-effect transistors (NW-FETs) ${ }^{1-9}$ hold great potential for detecting tiny amounts of charged biomolecules and are promising for applications in the area of bionanotechnology. While NWs are applied as NW-FET biosensors, NW is connected to two electrodes to form a current channel, with a gate electrode on the top or bottom of the NW to conduct gate voltage as the typical FET device. Because of ultrasensitive, label-free, and real-time response, the NW-FET biosensors can be used to detect DNA molecules, ${ }^{10-17} \mathrm{pH}^{18,19}$ gas molecules, ${ }^{20}$ proteins, ${ }^{21}$ and single virus particles. ${ }^{22}$

According to the principle of FETs, the gate voltage modulates the electron flow in the channel between the source and the drain. The nanowire acts as an electron bridge between the drain and the source. The identification of biotargets is based on a small change in induced electric signals through the environmental variation around the surface of the NW-FET device, where the gate voltage is applied in the subthreshold regime. ${ }^{23}$ Because of the difficulty of measuring small electrical signals (of the order under nanovolts or nanoamperes) and extracting data from the environmental disturbance mixed signals, appropriate electrical measurements and signal analysis methods are critical in biosensing experiments.

The accuracy of electrical measurements with FETs is affected by leakage current generated by the charging effect of a capacitor that appears across the gate oxide, in which the gate oxide serves as an insulator between gate and channel. This leakage current may significantly influence NW-FET because they operate in the femtoampere $(\mathrm{fA})$ to nanoampere $(\mathrm{nA})$ range. This paper reports a new method for NW-FET to avoid gate oxide current leakage problem. The proposed method uses a sloped variation in the gate voltage to reduce the potential difference between the gate and the source of an NW-FET. This leads to a more stable electrical characteristic measurement, and data obtained at different times after varying the gate voltage show the potential of using NW-FET in future applications. We verify by experiment that the proposed method achieves accurate results with stable performance.

\section{EXPERIMENTAL DEVICE AND METHODS}

The n-type polycrystalline silicon (poly-Si) NW-FETs are fabricated by a fully complementary metal-oxide-semiconductor-compatible (CMOS-compatible) process at the National Nano Device Laboratory in Hsinchu, Taiwan. The Si substrate consisted of a $100 \mathrm{~nm}$ silicon oxide $\left(\mathrm{SiO}_{2}\right)$ layer coated with a $50 \mathrm{~nm}$ silicon nitride $\left(\mathrm{Si}_{3} \mathrm{~N}_{4}\right)$ layer. A $1600 \mathrm{~nm}$ long, $500 \mathrm{~nm}$ wide $\mathrm{SiO}_{2}$ dummy structure was fabricated on the substrate. A $1000 \mathrm{~nm}$ thick $\alpha$-Si layer was deposited on the substrate and subjected to annealing at $600{ }^{\circ} \mathrm{C}$ to transform $\alpha$-Si into poly-Si.

Received: February 4, 2013

Revised: April 9, 2013

Published: April 10, 2013 
The poly-Si NWs with source and drain (S/D) contacts were then created using photolithography and plasma etching. The $\mathrm{S} / \mathrm{D}$ contacts were estivated by a $2000 \mathrm{~nm}$ thick $\mathrm{SiO}_{2}$ layer to prevent current leakage. ${ }^{18}$ Figure la shows our poly-Si NW

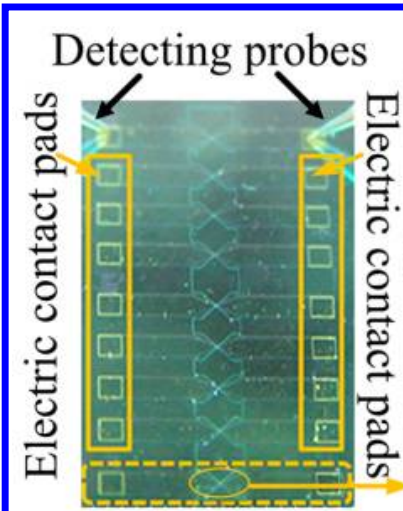

(a) Poly-Si NW-FET

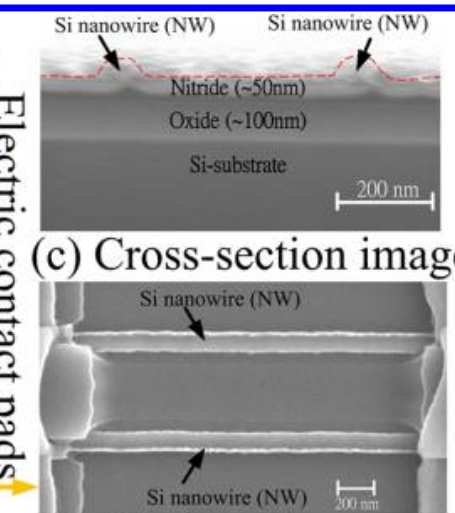

(b) SEM image
Figure 1. (a) Poly-Si NW-FETs with detecting probes. Inside the regions defined by an orange solid line and dashed line are the electric contact pads and one NW-FET device, respectively. The nanowire region is circled expanded as shown in (b). Top-view SEM image of a poly-Si NW-FET with a $200 \mathrm{~nm}$ scale bar. Cross-section structure of a poly-Si NW-FET device shown in (c). The nitride layer is $\sim 50 \mathrm{~nm}$, and the oxide layer is $\sim 100 \mathrm{~nm}$.

FETs and the electric contact pads in the orange line serve as sources and drains. Inside the orange dashed line is one of the NW-FET devices. In our measurement system, the signals from NW-FET device were collected by the two detecting probes. These two probes can be also easily moved to make different NW-FET measurements. Figure $1 \mathrm{~b}$ shows the SEM top-view image of a poly-Si NW structure which is consisted of two parallel poly-Si NW of $\sim 1600 \mathrm{~nm}$ length. The poly-Si NW-FET has two nanowires in each device; one of the NW is the dummy structure. The cross-section structure with NW-FET device is showed in Figure 1c.

In the NW-FET device, the current $I_{S}$ from the source terminal is based on the applied drain-to-source voltage $V_{\mathrm{DS}}$ and modified by the applied gate voltage $V_{\mathrm{GS}}$. All the measurements of the poly-Si NW-FETs were under backgating conditions in this study. Figure 2 shows $I_{\mathrm{S}}$ versus $V_{\mathrm{GS}}$ characteristics of a poly-Si NW-FET, for $V_{\mathrm{DS}}=0.1 \mathrm{~V}$ and $V_{\mathrm{GS}}$ varying from -1 to $3 \mathrm{~V}$ with a $0.2 \mathrm{~V}$ step. The electrical

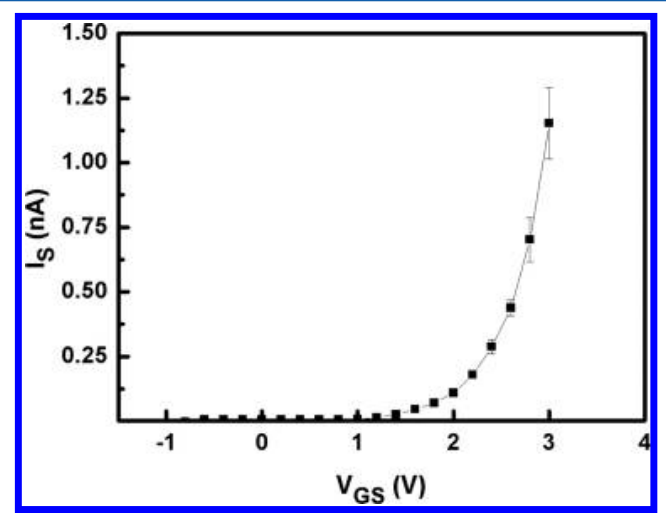

Figure 2. Measurement of current $I_{\mathrm{S}}$ with the gate-source voltage $V_{\mathrm{GS}}$ switched from -1 to $3 \mathrm{~V}$. properties of the poly-Si NW-FET exhibit threshold voltage of $V_{\text {th }} \sim 2.16 \mathrm{~V}$, subthreshold slope of $\mathrm{S} \sim 0.84 \mathrm{~V} /$ decade, and field effect mobility of $\mu \sim 225 \mathrm{~cm}^{2} / \mathrm{V}$ s. Figure 3 shows $I_{\mathrm{S}}-V_{\mathrm{DS}}$

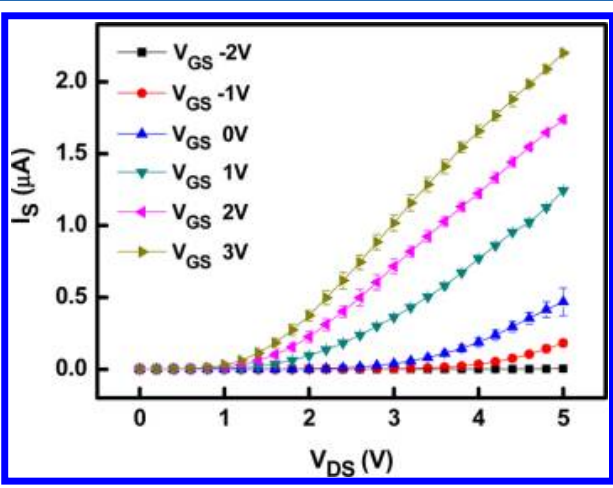

Figure 3. $I_{\mathrm{S}}$ vs $V_{\mathrm{DS}}$ measurement for various values of $V_{\mathrm{GS}}$.

curves for $V_{\mathrm{GS}}$ varying from -2 to $3 \mathrm{~V}$. The device exhibits typical metal-oxide-semiconductor field effect transistor (MOSFET) behavior, and the electronic properties of the poly-Si NW-FETs are well described by the conventional MOSFET theory. The experiments in this study reveal that current increases with increasing back-gate voltage $V_{\mathrm{GS}}$ superposed on forward $V_{\mathrm{DS}}$ in the NW-FET device. This characteristic also indicates the n-type-semiconductor nature of the device.

The measurement processes of the poly-Si NW-FETs were in an electromagnetic isolated probe station to reduce electromagnetic interference. All experimental results were obtained with measurement system included a high-precision source measure unit (PXI-4132, National Instrument) and a low-noise current preamplifier (SR570, Stanford Research Systems). These measurements of electric properties of the poly-Si NW-FETs were performed in air (room temperature, $45 \%$ humidity) under well-controlled conditions.

\section{RESULTS AND DISCUSSION}

The real-time responses of the source current $\left(I_{S}\right)$ of the poly-Si NW-FET was investigated in the atmosphere. The back-gate voltage $V_{\mathrm{GS}}$ was stepped up from 0 to $2.5 \mathrm{~V}$ in $0.5 \mathrm{~V}$ increments, with each step lasting $\sim 5 \mathrm{~s}$. During the step lasting time interval, $I_{S}$ was measured by our measurement system. The results described that $I_{\mathrm{S}}$ increased in conjunction with $V_{\mathrm{GS}}$, as shown in Figure 4 . When the $V_{\mathrm{GS}}$ was 0.5 and $1 \mathrm{~V}$, the original current $\left(I_{\mathrm{DS}}\right)$ was lower than the peak current and the

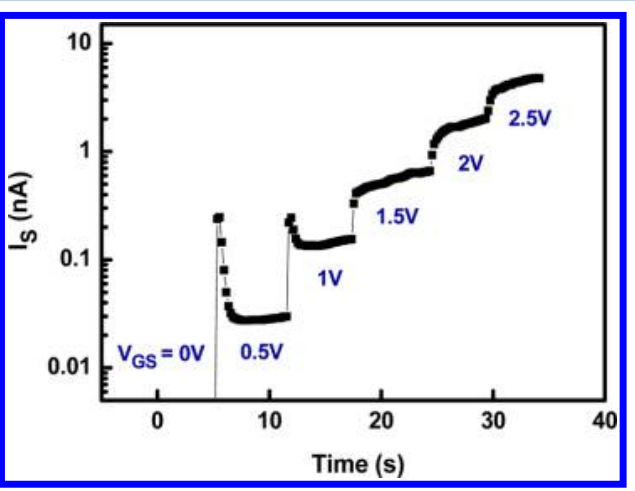

Figure 4. Temporal response of $I_{\mathrm{S}}$ as $V_{\mathrm{GS}}$ is stepped up from 0 to $2.5 \mathrm{~V}$ in $0.5 \mathrm{~V}$ increments. 
peak would be measured. As the $V_{\mathrm{GS}}$ was higher than $1.5 \mathrm{~V}$, the original current $\left(I_{\mathrm{DS}}\right)$ would cover the peak current and the $I_{\mathrm{DS}}$ current would make main contribution to data measurement. However, the spike of $I_{\mathrm{S}}$ were observed for the $V_{\mathrm{GS}}$ steps from 0 to $0.5 \mathrm{~V}$ and from 0.5 to $1 \mathrm{~V}$.

At the same time, the peaks of gate-to-source current $\left(I_{\mathrm{GS}}\right)$ in response to switching $V_{\mathrm{GS}}$ were also monitored in Figure 5. The

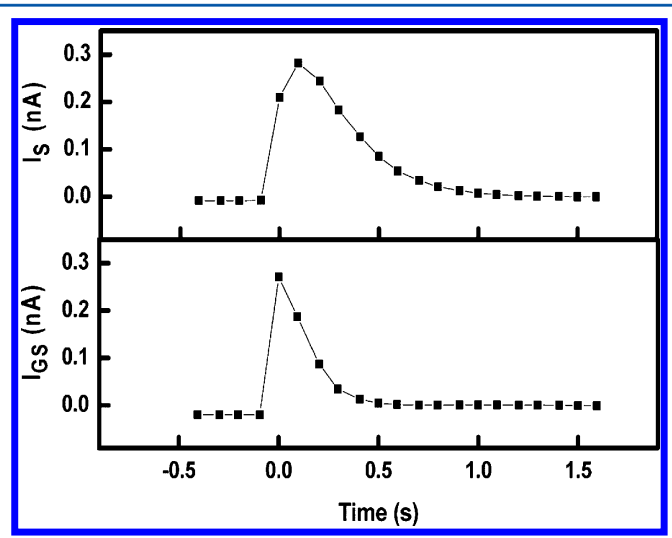

Figure 5. A comparison between $I_{\mathrm{S}}$ and $I_{\mathrm{GS}}$ after $V_{\mathrm{GS}}$ is switched from 0 to $0.5 \mathrm{~V}$.

peak of $I_{\mathrm{GS}}$ could be interpreted as minimal leakage current due to $V_{\mathrm{GS}}$ through the dielectric interface to the drain and source channel. Furthermore, the minimal leakage $\sim 0.3 \mathrm{nA}$ would cause $I_{S}$ bursting phenomenon at low gate voltage but would be stopped while gate voltage $V_{\mathrm{GS}}$ up to $1.5 \mathrm{~V}$. Although the leakage current from the back-gate voltage was covered for larger $I_{S}$, this event can affect the accuracy of poly-Si NW-FET measurements for biosensing applications because ultrahigh sensitivity is dependent on small electronic signal measurement.

The origin of this $I_{\mathrm{DS}}$ spike has been attributed to be the charging effect of a capacitor because the structure between source and gate in the poly-Si NW-FET is similar to a capacitor. For interpreting the bursting phenomenon clearly, a physical model, an equivalent transformation model of electric circuit of a n-type NW-FET, is given in Figure 6, where $\mathrm{G}$ is back gate, D is drain, $\mathrm{S}$ is source, $V_{\mathrm{GS}}$ is the back-gate voltage, $R_{\mathrm{GS}}$ is the equivalent resistance, $C_{\mathrm{GS}}$ is the equivalent capacitance, $g_{\mathrm{m}}$ is the transconductance of the device, $r_{\mathrm{o}}$ is the output impedance, and $V_{\mathrm{DS}}$ is the drain-to-source voltage. In the physical model, the drain-to-source current $\left(I_{\mathrm{DS}}\right)$ can be described by the MOSFET theory as follows ${ }^{21-24}$

$$
I_{\mathrm{DS}}=\mu_{n} C_{\mathrm{ox}}\left(\frac{W}{L}\right)\left(V_{\mathrm{GS}}-V_{\mathrm{th}}\right) V_{\mathrm{DS}} \approx g_{\mathrm{m}} V_{\mathrm{GS}}
$$

where $n$ is the charge-carrier effective mobility, $W$ is the gate width, $L$ is the gate length, $C_{\mathrm{ox}}$ is the gate oxide capacitance of the NW-FET unit area, and $V_{\text {th }}$ is the threshold voltage. The $V_{\mathrm{GS}}$ was applied to the $\mathrm{Si}$ substrate, which consisted of a silicon dioxide $\left(\mathrm{SiO}_{2}\right)$ layer and a silicon nitride $\left(\mathrm{Si}_{3} \mathrm{~N}_{4}\right)$ layer. Although no leakage current $I_{\mathrm{GS}}$ through the dielectric layers was expected, experimental results show that leakage current $I_{\mathrm{GS}}$ was nonzero during $V_{\mathrm{GS}}$ switching. Hence, these dielectric layers between source and gate can be described a simple series $R C$ circuit. To analyze this equivalent circuit quantitatively, Kirchhoff's loop rule is applied, and the response of $I_{\mathrm{GS}}$ is expressed as

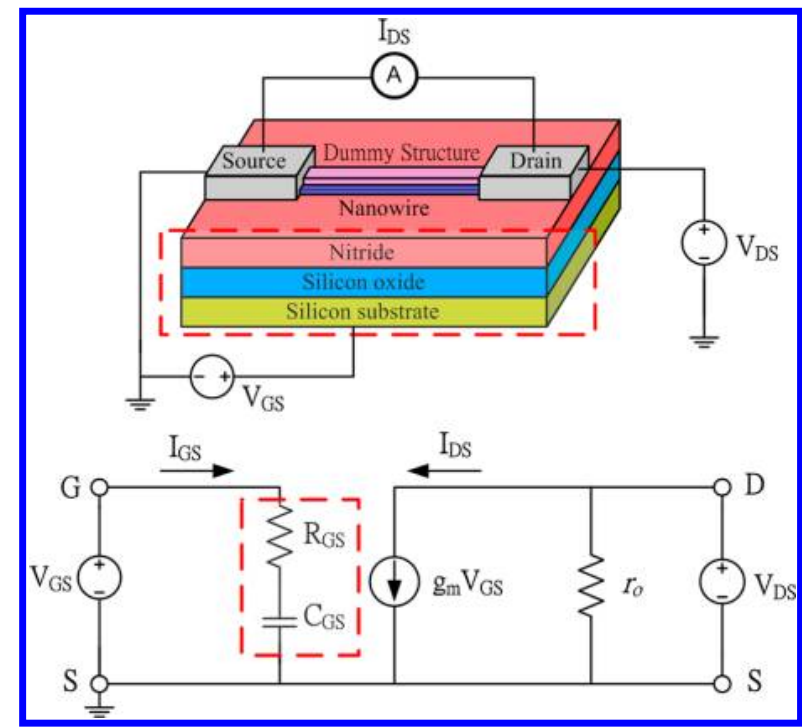

Figure 6. Equivalent circuit model of the NW-FET. $R_{\mathrm{GS}}$ and $C_{\mathrm{GS}}$ are the equivalent resistance and capacitance in the proposed physical model for the back-gate NW-FET structure, which is boxed in the red dashed line in the schematic above the circuit diagram. G is back gate, $\mathrm{D}$ is drain, $\mathrm{S}$ is source, $V_{\mathrm{GS}}$ is the back-gate voltage, $g_{\mathrm{m}}$ is the transconductance of the device, $r_{\mathrm{o}}$ is the output impedance, and $V_{\mathrm{DS}}$ is the drain-to-source voltage.

$$
I_{\mathrm{GS}}=\frac{V_{\mathrm{GS}}}{R_{\mathrm{GS}}}-\frac{q}{R_{\mathrm{GS}} C_{\mathrm{GS}}}
$$

where $I_{\mathrm{GS}}$ is replaced by $\mathrm{d} q / \mathrm{d} t$. Then $q(t)$ can be evaluated as

$$
q(t)=V_{\mathrm{GS}} C_{\mathrm{GS}}\left\{1-\exp \left[-t /\left(R_{\mathrm{GS}} C_{\mathrm{GS}}\right)\right]\right\}
$$

Then the current through the back gate substrate is obtained by

$$
I_{\mathrm{GS}}(t)=\frac{V_{\mathrm{GS}}}{R_{\mathrm{GS}}} \exp \left[-t /\left(R_{\mathrm{GS}} C_{\mathrm{GS}}\right)\right]
$$

The source current $I_{\mathrm{S}}(t)$ consists of two components. One is the leakage current $I_{\mathrm{GS}}(t)$ produced by the charging effect of a capacitor, and the other one is the drain-source current $\left(I_{\mathrm{DS}}\right)$ produced by the drain-to-source voltage $\left(V_{\mathrm{DS}}\right)$. The source current can be expressed as

$$
I_{\mathrm{S}}(t)=I_{\mathrm{GS}}+I_{\mathrm{DS}}=\frac{V_{\mathrm{GS}}}{R_{\mathrm{GS}}} \exp \left[-t /\left(R_{\mathrm{GS}} C_{\mathrm{GS}}\right)\right]+g_{\mathrm{m}} V_{\mathrm{GS}}
$$

This physical model indicates that current signal $I_{\mathrm{S}}$ will be contaminated with leakage current $I_{\mathrm{GS}}$ soon after $V_{\mathrm{GS}}$ is switched on or changed. The measured current signal $I_{S}$ would be dominated by real channel current between drain and source long after switching $V_{\mathrm{GS}}$. Typically, the efficiency of the leakage current would fade out while $t \gg R_{\mathrm{GS}} C_{\mathrm{GS}}$. Figure 7 shows that the experimental results are well reproduced by the simulation using the physical model outlined above; the correlation coefficient for the fit is $\sim 0.9354$. The experimental results fitted with eq 4 were extracted $R_{\mathrm{GS}} \approx 1.6 \mathrm{G} \Omega$ and $C_{\mathrm{GS}} \approx 0.27 \mathrm{pF}$ for $V_{\mathrm{GS}}$ switching from 0 to $0.5 \mathrm{~V}$ and with $V_{\mathrm{DS}}=0.1 \mathrm{~V}$. This physical model can therefore be used to explain the $I_{\mathrm{S}}$ spike phenomenon for measurements done while switching $V_{\mathrm{GS}}$ at low gate voltage.

According to previous discussions, the simplest method to avoid the interference of leakage current $\left(I_{\mathrm{GS}}\right)$ is applying appropriate time lag before collecting $I_{S}$ data in the biosensing 


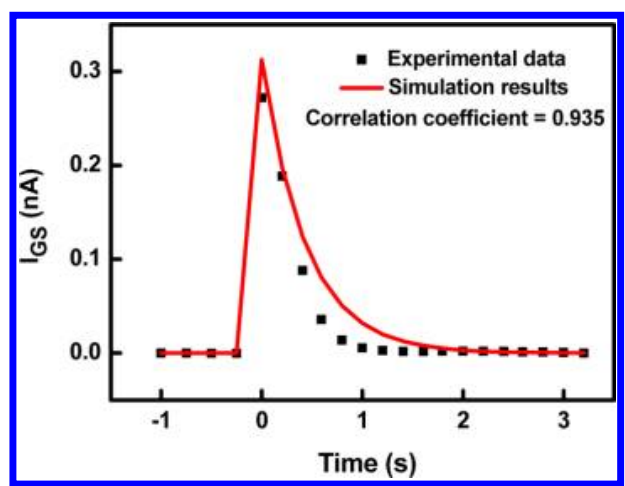

Figure 7. Experimentally measured $I_{\mathrm{GS}}$ for $V_{\mathrm{GS}}$ switched from 0 to 0.5 $\mathrm{V}$ state fit (red line) with eq 4.

experiment. However, equivalent resistance and capacitance of the NW-FET will differ after semiconductor processing; determining the required time lag to reach stable state of $I_{S}$ becomes difficult. To avoid the charging effect of the capacitance in the NW-FET, the sloped-gate voltage method was used to the $I_{S}$ measurement in Figure 8. On the basis of eq

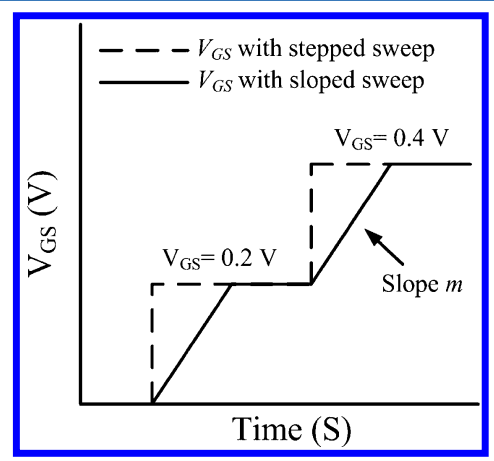

Figure 8. Slope modification used in $V_{\mathrm{GS}}$ switching from 0.2 to $0.4 \mathrm{~V}$.

4, $I_{\mathrm{GS}}$ is proportioned to $V_{\mathrm{GS}}$. In general, the $V_{\mathrm{GS}}$ sweep was applied a step function in $V_{\mathrm{GS}}$ switching (dotted line in Figure 8 ). Here the step $V_{\mathrm{GS}}$ in common use was modified as a sloped $V_{\mathrm{GS}}$ changing (solid line in Figure 8) to reduce the gate voltage different under $\Delta t$. Therefore, $V_{\mathrm{GS}}$ was slowly stepped up to measure $I_{S}$ independently of $V_{\mathrm{GS}}$. The applied potential difference between the poly-Si NW-FET source and gate was sequentially increased $V_{\mathrm{GS}}$ to the experimentally required value.

NW-FET devices are usually used to detect biosignal variances in a liquid environment. Therefore, the experiments of $I_{S}$ measurement results compared without sloped-gate voltage method to with the method in $\mathrm{PB}$ (phosphate buffer, $10 \mathrm{mM}$ ) solution in Figure 9. After $V_{\mathrm{GS}}$ was switched from 0.2 to $0.4 \mathrm{~V}$, the results shown in Figure 9a were acquired without adjusting the $V_{\mathrm{GS}}$ slope $m$ (step function), and Figure $9 \mathrm{~b}$ shows results for $I_{S}$ with the slope adjusted to $m=0.04$. The corresponding variances $\sigma$ of different operating conditions in $I_{S}$ are $1.12 \times 10^{-10}$ and $1.783 \times 10^{-11}$, respectively. The corresponding variances $\sigma$ is defined by the standard deviation of the averaged $I_{\mathrm{S}}$ to interpret the variance of measurement of $I_{S}$. The $\sigma$ is represented by $(6)$ as

$$
\sigma=\sqrt{\frac{1}{N} \sum_{i=1}^{N}\left(X_{\text {time }}-\mu_{\text {time }}\right)}
$$

where

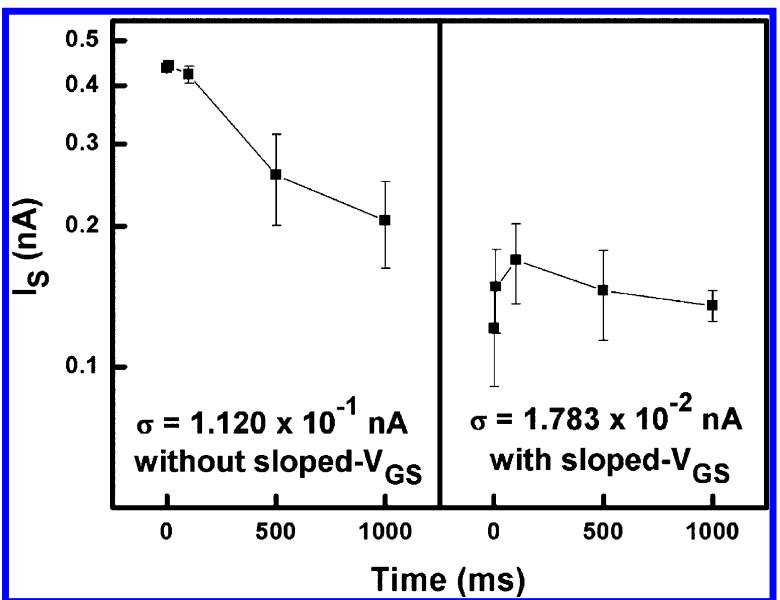

Figure 9. (a) $I_{\mathrm{S}}$ measured at various times after switching $V_{\mathrm{GS}}$ from 0.2 to $0.4 \mathrm{~V}$ without sloped-gate method in PB solution. (b) Same measurement as for (a) but with sloped-gate voltage method.

$$
\mu=\frac{1}{N} \sum_{i=1}^{N} X_{\text {time }}
$$

Time is five different sampling points of time $(1,10,100,500$, and $1000 \mathrm{~ms}$ ). These results indicate that switching $V_{\mathrm{GS}}$ with an adjusted slope $m$ permits simultaneous measurements of $I_{\mathrm{S}}$ that are unaffected by the leakage current $I_{\mathrm{GS}}$. The results of the variances for $I_{\mathrm{S}}$ are summarized in Table 1 .

Table 1. Variance in Measured $I_{\mathrm{DS}}$ Values (ampere) after Switching $V_{\mathrm{GS}}$ with and without Adjusting $V_{\mathrm{GS}}$ Slope in Three Experiment Conditions

\begin{tabular}{ccc}
\multicolumn{3}{c}{ variance of $I_{\mathrm{DS}}(\mathrm{A})$ with different time intervals } \\
\hline experiment condition & without sloped $V_{\mathrm{GS}}$ & with sloped $V_{\mathrm{GS}}$ \\
air & $1.189 \times 10^{-10}$ & $1.985 \times 10^{-12}$ \\
DI water & $1.788 \times 10^{-10}$ & $1.120 \times 10^{-10}$ \\
$\mathrm{~PB}$ & $2.682 \times 10^{-11}$ & $1.783 \times 10^{-11}$ \\
\hline
\end{tabular}

For the poly-Si NW-FET in air, the variance reduced almost 2 orders of magnitude upon using the sloped-gate voltage method. Under liquid (De Ion water, DI Water) conditions, the variance decreased slightly more than 1 order of magnitude. The variance decreased $\sim 1$ order of magnitude in PB solution. These data demonstrate that the sloped-gate voltage method can also be applied under liquid conditions, making it a promising technique for future in biosensing processing. In addition, the slope-gate voltage method on reproducibility has been shown in the Supporting Information.

\section{CONCLUSION}

NW-FET has become an import device in the area of biological sensing application. The detecting tiny amounts of charged biomolecules affect femtoampere (fA) to nanoampere ( $\mathrm{nA}$ ) current change of electrical characteristic. Experimental results show that the leakage current $I_{\mathrm{GS}}$ is caused by the charging effect of an equivalent capacitor in the NW-FET structure. To explain this phenomenon, a physical model with equivalent resistance and capacitance was developed to simulate the leakage current of the poly-Si NW-FET, and the fit based on this model has a high correlation with the experimental data. This study also shows that the leakage current $\left(I_{\mathrm{GS}}\right)$ affects the 
accuracy of NW-FET measurements. To solve this problem, a novel $V_{\mathrm{GS}}$ switching method, which is the sloped-gate method, was proposed. The results indicate that the sloped-gate method decreases the variance in $I_{\mathrm{DS}}$ measured after switching $V_{\mathrm{GS}}$, for poly-Si NW-FETs detections in air, liquid, and phosphate buffered solution (PBS). This method of measuring $I_{S}$ in NWFETs can be used for accurate measurements of different targets or for other applications as part of a biosensor platform; on the other hand, the physical model is able to evaluate the equivalent capacitor in the NW-FET for other analysis parameters of the biosensing application.

\section{ASSOCIATED CONTENT}

\section{S Supporting Information}

Table I. This material is available free of charge via the Internet at http://pubs.acs.org.

\section{AUTHOR INFORMATION}

\section{Corresponding Author}

*E-mail ysyang@faculty.nctu.edu.tw, Tel 886-3-5729287 (Y.S.Y.); e-mail chhwang@itrc.narl.org.tw, Tel 886-3-5779911 (C.H.H.).

Notes

The authors declare no competing financial interest.

\section{ACKNOWLEDGMENTS}

The NW-FET devices were prepared at National Nano Device Laboratories, National Applied Research Laboratories, Taiwan. We thank Professor Chii-Dong Chen of Academia Sinica, Taiwan, for helpful discussions. This work was supported by National Science Council (NSC101-2923-B-009-001-MY3 and 101-2622-B-009-001-CC1) and MOE-ATU Program.

\section{REFERENCES}

(1) Cui, Y.; Duan, X.; Hu, J.; Lieber, C. M. Doping and Electrical Transport in Silicon Nanowires. J. Phys. Chem. B 2000, 104, 52135216.

(2) Cui, Y.; Wei, Q.; Park, H.; Lieber, C. M. Functional Nanoscale Electronic Devices Assembled using Silicon Nanowire Building Blocks. Science 2001, 293, 1289-1292.

(3) Duan, X.; Huang, Y.; Cui, Y.; Wang, J. F.; Lieber, C. M. Indium Phosphide Nanowires as Building Blocks for Nanoscale Electronic and Optoelectronic Devices. Nature 2001, 409, 66-68.

(4) Huang, Y.; Duan, X.; Cui, Y.; Lauhon, L. J.; Kim, K. H.; Lieber, C. $\mathrm{M}$. Logic Gates and Computation from Assembled Nanowire Building Blocks. Science 2001, 294, 1313-1317.

(5) Huang, Y.; Duan, X.; Cui, Y.; Lieber, C. M. Gallium Nitride Nanowire Nanodevices. Nano Lett. 2002, 2, 101-104.

(6) Duan, X.; Huang, Y.; Lieber, C. M. Nonvolatile Memory and Programmable Logic from Molecule-Gated Nanowires. Nano Lett. 2002, 2, 487-590.

(7) He, H., Jr.; Zhang, Yi Y.; Liu, J.; Moore, D.; Bao, G.; Wang, Z. L. ZnS/Silica Nanocable Field Effect Transistors as Biological and Chemical Nanosensors. J. Phys. Chem. C 2007, 111, 12152-12156.

(8) Varsano, D.; Garbesi, A.; Felice, R. D. Ab Initio Optical Absorption Spectra of Size-Expanded xDNA Bases. J. Phys. Chem. B 2007, 111, 14012-14021.

(9) Hsiao, C.-Y.; Lin, C.-H.; Hung, C.-H.; Su, C.-J.; Lo, Y.-R.; Lee, C.-C.; Lin, H.-C.; Ko, F.-H.; Huang, T.-Y.; Yang, Y.-S. Poly-silicon Nanowire Field Effect Transistor for Biosensing Application. Biosens. Bioelectron. 2009, 24, 1223-1229.

(10) Jones, P. A.; Baylin, S. B. The Epigenomics of Cancer. Cell 2007, $128,683-692$.
(11) Patolsky, F.; Timko, B. P.; Zheng, G. F.; Lieber, C. M. Nanowire-Based Nanoelectronic Devices in the Life Sciences. MRS Bull. 2007, 32, 142-149.

(12) Yang, M.; Qu, F.; Lu, Y.; He, Y.; Shen, G.; Yu, R. Platinum Nanowire Nanoelectrode Array for the Fabrication of Biosensors. Biomaterials 2006, 27, 5944-5950.

(13) Hahm, J.; Lieber, C. M. Direct Ultrasensitive Electrical Detection of DNA and DNA Sequence Variations Using Nanowire Nanosensors. Nano Lett. 2004, 4, 51-54.

(14) Lin, C.-H.; Hung, C.-H.; Hsiao, C.-Y.; Lin, H.-C.; Ko, F.-H.; Yang, Y.-S. Poly-Silicon Nanowire Field-Effect Transistor for Ultrasensitive and Label-Free Detection of Pathogenic Avian Influenza DNA. Biosens. Bioelectron. 2009, 24, 3019-3024.

(15) Gao, X. P.; Zheng, G.; Lieber, C. M. Subthreshold Regime Has the Optimal Sensitivity for Nanowire FET Biosensors. Nano Lett. 2010, 10, 547-552.

(16) McAlpine, M. C.; Ahmad, H.; Wang, D.; Heath, J. R. Highly Ordered Nanowire Arrays on Plastic Substrates for Ultrasensitive Flexible Chemical Sensors. Nat. Mater. 2007, 6, 379-384.

(17) Stern, E.; Klemic, J. F.; Routenberg, D. A.; Wyrembak, P. N.; Turner-Evans, D. B.; Hamilton, A. D.; LaVan, D. A.; Fahmy, T. M.; Reed, M. A. Label-Free Immunodetection with CMOS-Compatible Semiconducting Nanowires. Nature 2007, 445, 519-522.

(18) Patolsky, F.; Zheng, G.; Hayden, O.; Lakadamyali, M.; Zhuang, X.; Lieber, C. M. Electrical Detection of Single Viruses. Proc. Natl. Acad. Sci. U. S. A. 2004, 101, 14017-14022.

(19) Lu, M.-P.; Hsiao, C.-Y.; Lai, W.-T.; Yang, Y.-S. Probing the Sensitivity of Nanowire-Based Biosensors Using Liquid-Gating. Nanotechnology 2010, 21, 425505-1-5.

(20) Lu, M.-P.; Hsiao, C.-Y.; Lo, P.-Y.; Wei, J.-H.; Yang, Y.-S.; Chen, M.-J. Semiconducting Single-Walled Carbon Nanotubes Exposed to Distilled Water and Aqueous Solution: Electrical Measurement and Theoretical Calculation. Appl. Phys. Lett. 2006, 88, 053114-1-3.

(21) Ishikawa, F. N.; Curreli, M.; Chang, H.-K.; Chen, P.-C.; Zhang, R.; Cote, R. J.; Thompson, M. E.; Zhou, C. A Calibration Method for Nanowire Biosensors to Suppress Device-to-Device Variation. ACS Nano 2009, 3, 3969-3976.

(22) Galup-Montoro, C.; Schneider, M. C. MOSFET Modeling for Circuit Analysis and Design; World Scientific: London, 2007.

(23) Malik, N. R. Electronic Circuits: Analysis, Simulation, and Design; Prentice Hall: Englewood Cliffs, NJ, 1995.

(24) Wang, J.; Gudiksen, M. S.; Duan, X.; Cui, Y.; Lieber, C. M. Highly Polarized Photoluminescence and Photodetection from Single Indium Phosphide Nanowires. Science 2001, 293, 1455-1457. 